\title{
Living with the challenges of treating tuberculosis
}

\section{The patient and healthcare professional perspective}

\section{Patient perspective}

The father of Alessandro, an 11 year old boy with multidrug-resistant tuberculosis

As parents, we found it very difficult to deal with the fact that our child had tuberculosis (TB). It was not just the shocking diagnosis, but the whole experience involving treatment and medical examinations is one we would rather leave behind us.

Our son had been ill for 5 months before we were able to get a diagnosis of TB for him and we feel that a deeper knowledge among healthcare professionals could have helped us to make this process a lot shorter. Of course, like in many other European countries, TB is thought to have been eradicated in Italy, and the lack of awareness about its re-emergence contributed, in our view, hugely to the delay in diagnosis.

Still, the diagnosis was to be only the beginning. Our son spent 7 months on an isolation ward in hospital. We had to consent to medical examinations, some of which were invasive and caused him a lot of pain. This was not the only stress we experienced. Living on an isolation ward meant a loss of his social environment, and there were many tears about being deprived of friends and family.

As our child had to deal with the physical, social and emotional effects of his illness, we struggled trying to balance our work and home life against being with him at hospital. It was incredibly difficult in Italy to wade through the bureaucracy and get the assistance that we, as a family, needed to support and look after our severely ill child in hospital.
We also feel that a lack of specialists should be addressed by our government, as well as the time it took to get the drugs for his treatment imported.

Still, in spite of these difficulties, there are also many thanks we need to give to the people involved in our son's treatment and care. Not only did they take care of our son, but they also managed to get him a laptop, which eventually enabled him to catch up with vital school work and which alleviated his social isolation.

"When I was hospitalised, I was very sad because I was in isolation and had no contact with my friends, my sister, my brother and also my cat. However, the team of paediatricians, led by Susanna Esposito, looked after me in a professional but also lovely way. They always tried to encourage me, even in the worse moments. They told me it was possible to cure my illness and they organised a school programme in the hospital for me. When I was discharged after 6 months of hospitalisation, I was happy and grateful to the paediatricians and nurses who took care of me."

Alessandro

\section{The healthcare professional's perspective}

Professor Susanna Esposito is Professor of Paediatrics at The University of Milan, Director of the Paediatric Highly Intensive Care Unit at Fondazione IRCCS Ca' Granda 
Ospedale Maggiore Policlinico, Milan, Italy and President of the World Association of Infectious Diseases and Immunological Disorders (WAIDID) and the Italian Society for Paediatric Infectious Diseases (SITIP)

My main research interest and area of clinical practice since 1995 has been paediatric infectious diseases. Over the last five or so years, we have seen an increase in the number of complicated TB cases. TB is interesting as it is a respiratory tract infection, and it is quite common for patients to come to us from other hospitals having experienced a delay with diagnosis.

Alessandro was initially seen by an ear nose and throat (ENT) specialist because he had laryngitis with dysphonia, impairment of the voice. After looking at the images captured during an endoscopy, the specialist asked for a consultation with us as experts in infectious diseases. As soon as we saw Alessandro, we performed a chest radiograph, which showed that he had TB.

On the whole, Alessandro's symptoms were ENT-related rather than pulmonary. As well as dysphonia, his parents told us that he was experiencing light asthma, lethargy and hearing loss. He didn't have a history of fever or cough.

The tuberculin skin test (TST) was negative but an interferon- $\gamma$ release assay (IGRA) confirmed our suspicions of TB. We also examined his gastric aspirates and found Mycobacterium tuberculosis.

Treatment was quite complicated. Initially, we started Alessandro on a course of four drugs, but tests showed that his gastric aspirates were still positive for TB. When we had his culture results 3 weeks later, we discovered that he was resistant to all of the firstand second-line drugs. I was very worried, as Alessandro's condition was deteriorating, so I contacted the European Respiratory Society (ERS)/World Health Organization (WHO) TB Consilium about treatment options, specifically two new drugs, delamanid and bedaquiline. Both the regional health authorities and drug companies told us that it would not be possible to obtain these drugs, however the Consilium affirmed that either drug would be mandatory in the drug regime. This recommendation from experts in multidrug-resistant (MDR) TB and extremely drug-resistant (XDR) TB enabled me to push the companies to provide the drugs directly if I could get approval from my ethics committee and the relevant authorities in Italy. I managed to get both, and so was able to get delamanid for Alessandro.
After beginning this treatment, Alessandro's gastric aspirates were negative after 7 days. $\mathrm{He}$ was feeling better and was able to eat, and was showing improvement in all systemic parameters. Alessandro was taking five different drugs for about 2 months. The treatment was difficult: Alessandro lost weight, going from $42 \mathrm{~kg}$ to $38 \mathrm{~kg}$ in a month, was very tired and had anaemia, among other problems.

Alessandro was quite shy, so he found it difficult to explain how he was feeling during the worst months, when his TB was active. After this phase he was much happier, and we started teaching him in the hospital to stop him falling behind at school, as he missed 9 months of classes while he was in hospital. During this time, he wrote a lovely letter about his experience and his relationship with his doctors and nurses.

Alessandro's case is quite interesting as he and his family are from Italy, a non-endemic country, with no history of foreign travel. His father was negative for TB, but his mother and siblings had latent TB.

The lesson to be learned here is that each time we have respiratory infections not responsive to standard treatment and with persistent symptoms we should first rule out the possibility of having TB. Treatment for TB can be 6 or 9 months, or even longer if necessary, so it is very important to point out to people newly diagnosed with TB and their families that they need to complete treatment in order to avoid complications and drug resistance. After diagnosis, it is vital to be seen regularly and monitored by a doctor over a long period during treatment.

One of the challenges of treating children with TB is the availability of off-label drugs, due to their age. Furthermore, there are very few drugs available in syrup form for children that are not able to swallow pills. Another problem is that certain drugs are not available in each country, so we need adequate importation procedures that will not take too long, especially during holiday periods. For example, when we were treating Alessandro, there was an instance when his drugs had not been imported from the UK to Italy on time.

My hope is that the diagnosis of TB will improve, especially in paediatrics, and that tests can be available in a few hours. I hope that, with new drugs and early diagnosis, we can try to avoid the complications that we had with Alessandro. 